\title{
Using the Web for Collaborative Language Learning and Teaching
}

\author{
Werner Winiwarter \\ University of Vienna \\ Research Group Data Analytics and Computing \\ Universitätsstraße 5, Vienna, A-1010 Austria \\ werner.winiwarter@univie.ac.at
}

\begin{abstract}
For the past few years, the research focus in ComputerAssisted Language Learning has shifted towards learner-centered approaches, in particular through Web-based source material and linguistic resources. Recent findings emphasize the importance of personalizing the learning experience, integrating it seamlessly into everyday activities, and making use of collaborative and social features.

We addressed these issues by developing COLLIE, a COllaborative Language Learning and Instruction Environment. We have implemented a first prototype for Japanese, which lets the students work with Japanese Web documents and offers appealing visualizations of linguistic information at the character, word, and sentence level. For each user, we store all the relevant information about personal preferences to customize the display of the Web content and about the interaction with COLLIE as valuable input for the instructor.

A key component of COLLIE is the machine translation system JET$C A T$, which has the big advantage that it learns translation rules automatically from bilingual corpora. The acquisition process is performed by parsing source and target sentences, retrieving word translations of Japanese content words from lexical resources, mapping the content words to corresponding English words through an iterative contextual disambiguation process, and, finally, structurally aligning the two trees based on the word alignment data.

The translation rules used in our formalism can be easily interpreted and understood by language students. Therefore, we created an animated view of the source and target elements of the individual rules in the Japanese and English parse trees to convey a better understanding of the translation process.

The JETCAT rule base can be incrementally updated by simply correcting translation results. We provide this feature for the language students so that they can create their own personal translation rule bases and customize COLLIE according to their preferences. In addition, we offer the possibility to display a dynamically generated list of the most popular translations produced with the rule bases of the other COLLIE users.

We finally report on new directions and activities towards terminology discovery, semantic annotation and retrieval of multimedia documents, community building, mobile language learning, and language learning in collaborative $3 \mathrm{D}$ virtual worlds.
\end{abstract}

